\title{
Albrecht von Haller und der praktische Arzt seiner Zeit
}

Von Urs Boschung

\section{Einleitung: Haller als Praktiker}

Anstoß zur Beschäftigung mit Hallers Tätigkeit als praktischer Arzt gab Erich Hintzsches Edition der Briefe Hallers an den Lausanner Kollegen Tissot, den berühmten Praktiker, mit dem sich Haller sehr oft über Fragen der praktischen Medizin und auch über gemeinsame Patienten unterhalten hat ${ }^{1}$. Hallers Praxis war bisher wenig beachtet worden, und auch bei seinen Zeitgenossen war offenbar wenig darüber bekannt. Die Behauptung eines Nachrufs von 1778: «Er hat die Arzneikunst niemals ausgeübet ...» weist Johann Georg Zimmermann entschieden zurück ${ }^{2}$ : «Haller hat die Arzneikunst allerdings ausgeübet, und zwar in Bern, von Haus zu Haus, von 1729 bis 1736. In Göttingen lies er sich von 1737 bis 1753 oft aus Freundschaft, oder in wichtigen Fällen, zu Kranken rufen. Er that eben das nachher, zu jeder Zeit, in Bern. Schriftlich hat er seine Räthe immer ertheilt, und für den gemeinen Mann in der Schweiz, wie an viele große Herren von Europa. Über die dunkelsten Krankheiten fragten ihn sehr oft Ärzte um seinen Rath. Die Kranken seiner Familie hat er immer besorget ... .» Haller war aber zweifellos nicht der geborene Praktiker, auch dies belegen seine Briefe an Tissot, wo es bezeichnenderweise heißt ${ }^{3}$ : «Dieu me preserve de Pratique, je n'y saurois tenir. J'aime mieux m'aveugler avec le microscope au fort du soleil sur un œil de poisson.» Immerhin war er nicht ein weltfremder Theoretiker, wenn er sich zur «ausübenden Heilkunde» und zur Stellung ihrer Vertreter geäußert hat. Wir können daraus einigen Aufschluß erwarten über jene Leitbilder der Zeit, die für einen erfahrenen akademischen Lehrer, für den Assessor perpetuus des bernischen Sanitätsrates maßgebend waren, maßgebend für die Ausbildung des Nachwuchses und für die Verbesserung der Medizinalgesetzgebung.

\section{Zweierlei Praxis}

Hallers Urteil über zwei prominente Praktiker, die beide auf dem Boden der bernischen Republik tätig waren, soll uns den Einstieg in die Thematik 
erleichtern. Über Tissot, den bekannten Lausanner Arzt, schrieb er in einer Rezension 1761-624: «Hr. Tissot ist ein beliebter und stark gebrauchter Arzt, der zwar zu Montpelier seine ersten Gründe gelegt, in den besten Schriften aber, und zumal in der Natur selber sich seit dem nüzlich unterrichtet hat.» Tissot ist also der typische akademisch gebildete Arzt, der im Ausland studiert hat, in Montpellier zwar, einer in Hallers Augen nicht sehr angesehenen Universität, der sich seither durch Lektüre der neueren medizinischen und physiologischen Literatur, zumal der Schriften Hallers weitergebildet und eine vielfältige Erfahrung als Praktiker gesammelt hat.

Als Gegenstück hier das Urteil Hallers über einen nicht minder bekannten Praktiker, nämlich Michel Schüppach aus dem Jahre $1773^{5}$ : «Il n’a ni etudié, ni connu d'anatomie, ni quité son village.» Der Wundarzt oder Chirurg, den Michel Schüppach darstellt, kommt also bei Haller ziemlich schlecht weg. Er hat kein reguläres Studium absolviert, er verfügt über keine anatomischen Kenntnisse, war nie im Ausland, kennt die großen Spitäler der Weltstädte nicht, ist bloß ein Dorfarzt ohne Weltläufigkeit.

Um nun auf diese positiv und negativ bewerteten Punkte näher eintreten zu können, ist es notwendig, die Situation der praktischen Medizin, wie sie sich Haller darbot, kurz zu skizzieren ${ }^{6}$.

Wie aus der Gegenüberstellung von Tissot und Schüppach hervorgeht, ist die Heilkunde immer noch gespalten in innere Medizin und Chirurgie. Haller findet diese Trennung nicht durch die Sache selbst, sondern höchstens durch die Beschränktheit des menschlichen Verstandes gerechtfertigt. Die Chirurgie, besonders die operative Chirurgie, hat große Fortschritte verzeichnet, und in Frankreich haben die Chirurgen einen bemerkenswerten sozialen Aufstieg vollzogen. Dagegen ist die innere Medizin, was Diagnostik (Zeichenlehre) und Prognostik betrifft, kaum über Hippokrates hinausgekommen, erst Sydenham hat dessen Betrachtungsweise wieder aufgenommen. Das therapeutische Arsenal immerhin ist wesentlich reicher und schlagkräftiger geworden, man denke an Chinarinde und Mineralsäuren, die zuverlässig gegen Fieber und Fäulnis wirken; Haller mißt sich selbst einiges Verdienst zu an deren Einführung und Bekanntmachung?

Sehr vieles bedarf allerdings der Vervollkommnung. Der Weg dazu führt über die pathologische Anatomie und das klinische Experiment im Spital. Früher verbot der Aberglaube die Leichenöffnung. Heute aber steht der Weg frei «a cette science admirable des rapports des signes et des symptomes observés dans le malade pendant ses souffrances, avec le véritable siège de son mal, et avec la dégénération de la machine animale qui a produit ces 
symptomes. C'est l'anatomie pratique seule qui peut nous procurer une pathologie digne de notre confiance» ${ }^{8}$. Mit der Erforschung der Zusammenhänge zwischen Klinik und Anatomie einhergehen muß die Evaluierung zuverlässiger Arzneimittel. Dazu eignet sich am besten die Vorprüfung im Tierversuch und die kontrollierte Anwendung unter Spitalbedingungen. Gerade in diesem Punkt, glaubt Haller, ist das 18. Jahrhundert einen Schritt weiter gekommen.

Die Praxis ist der Mittelpunkt der Medizin; hier haben sich alle Spezialkenntnisse zu vereinigen ${ }^{9}$. Wie aber muß der medizinische Unterricht beschaffen sein, damit dies gewährleistet ist?

Die Zielsetzung für die beiden Hauptklassen der Praktiker, Ärzte und Chirurgen, war von alters her ungleich. Der Wundarzt durfte und sollte sich mit den Grundzügen der operativen und medikamentösen Behandlung der äußeren Krankheiten begnügen. Dagegen wurde vom Arzt ein umfassendes medizinisches Wissen erwartet sowie die Fähigkeit, die «Diener der Kunst» (Chirurgen, Hebammen, Apotheker) sinnvoll einzusetzen. Diese Aufteilung der Tätigkeitsbereiche war aber im 18.Jahrhundert längst nicht mehr überall gültig und wohl auch in früheren Jahrhunderten nur teilweise durchgesetzt worden. So gab es Ärzte wie Lorenz Heister, die selbst operierten und, der Zahl nach viel wichtiger, Chirurgen, die auch innere Krankheiten behandelten. Auf dem Lande war ja der Wundarzt meist ohne Aufsicht und Beratung der Arztes, der es vorzog, sich in der Stadt niederzulassen.

\section{Bildungsziele des akademischen Lehrers}

Betrachten wir zuerst Hallers Beitrag zur Ausbildung des Arztes. Während siebzehn Jahren lehrt er an der Universität Göttingen die Fächer Anatomie, Botanik und Chirurgie. Seine wissenschaftliche Arbeit gilt der Anatomie und Physiologie (der «Animata anatome») und der Botanik. Letztere betreibt er um ihrer selbst willen, als biologische Disziplin, und nicht als Hilfsfach für den Mediziner. Anatomie und Physiologie dagegen werden zu eigentlichen Grundlagen der Medizin, dies ist Hallers besondere Leistung für Göttingen. Die Anatomie, und mit ihr die Physiologie, ist das einzige Fach, das über ein eigenes Gebäude verfügt, ein Gebäude, das dem Unterricht und der Forschung dient. Der Student wohnt hier nicht wie an anderen Universitäten hie und da einer anatomischen Demonstration bei, sondern hat 
Gelegenheit, eigenhändig zu präparieren. Mehr noch, er nimmt an der eigentlichen Forschungsarbeit teil, indem ihm Haller die Bearbeitung einer speziellen Frage überträgt und diese zu einer Dissertation ausarbeiten läßt. So hat Johann Georg Zimmermann, später Stadtarzt in Brugg und Hannoverscher Leibarzt, für seine Doktorarbeit eine große Zahl von Tierexperimenten zur Begründung der Hallerschen Irritabilitätslehre durchgeführt.

Haller wollte beim Studenten aber nicht nur den Forschergeist wecken, er wollte ihm auch das gesicherte Wissen in handlicher Form anbieten. So entstanden die Primae lineae physiologiae (1747), Grundzüge der Physiologie, die mehrere Auflagen erlebten und vielerorts zur Grundlage der Vorlesung dienten.

Im Auftrag des Professors der Anatomie lag es, dem Medizinstudenten die Theorie der Chirurgie und die Grundlagen der operativen Technik zu vermitteln. Wie nicht wenige seiner Kollegen beschränkte sich Haller darauf, die wichtigsten Operationen an der Leiche vorzuzeigen. Daß der Student damit nur mangelhaft auf die Praxis vorbereitet wurde, war er sich wohl bewußt. Die wichtigste Voraussetzung zur Verbesserung des medizinischen Unterrichts war zweifellos die Errichtung eines Spitals. Dieses fehlte in Göttingen wie in mancher anderen deutschen Universitätsstadt, und zwar bis gegen Ende des Jahrhunderts, obschon sich Haller in seinen letzten Jahren sehr dafür eingesetzt hat. Mehr Erfolg war ihm auf dem Gebiet der Geburtshilfe beschieden, wo sein Schüler Johann Georg Roederer zum ersten Professor dieses Faches an einer deutschen Universität ernannt wurde.

In Göttingen war man sich bei der Gründung der Universität schon 1733 im klaren, daß der medizinische Unterricht praktisch und anschaulich zu erfolgen hatte, «denn der professor mag lehren, was er will: wenn er nichts zeigen kan, so gehen die studenten hin, wo sie Kranckheiten und Curen selbst zu sehen bekommen.» ${ }^{10}$ Bis aber aufklärerischer Verbesserungseifer dieses Postulat im Laufe des Jahrhunderts erfüllt hatte, war der Student gezwungen, seine Kenntnisse auf langen Reisen mühsam zusammenzusuchen. Die «peregrinatio academica» war indessen nicht nur eine lästige Pflichtübung. Sie entsprach dem Drang der Jugend nach Bewegung und Abwechslung, gab Gelegenheit, die großen Männer des Faches persönlich kennenzulernen, und hob das Ansehen des Reisenden. Der Mediziner im besonderen mochte in den Spitälern der großen Städte Kranke von Fleisch und Blut sehen, den Visiten der Ärzte und Chirurgen folgen, den Operationen beiwohnen und in Sektions-, Operations- und Geburtshilfekursen selbst Hand anlegen ${ }^{11}$. Manches, was in der Fremde als nützlich erfahren worden war, wurde später zu Hause verwirklicht, wie sich auch am Beispiel Hallers zeigen ließe. 


\section{Sanitätsrat Hallers Reformvorschläge}

Ursprünglich zur Seuchenabwehr bestimmte Behörden übernahmen im Laufe des 18. Jahrhunderts besonders in monarchischen Staaten in zunehmendem Maße die Kontrolle über das Medizinalwesen. Man reglementierte die Ausbildung, erklärte Stundenpläne und Curricula für verbindlich und führte Prüfungen ein, deren Bestehen für die Zulassung zur Praxis unerläßlich waren. In Bern hieß diese Behörde Sanitätsrat. Haller gehörte ihm von 1756-1758 und 1765-1777 an ${ }^{12}$. Als Mitglied dieses Rats verfaßte er 1765 ein ausführliches Gutachten: «Gedanken über die Verbesserung der zur Kunst zu Heilen einschlagenden Wissenschaften.» ${ }^{13}$ Hier zeigt er die Möglichkeiten der Republik auf, die Qualität der Medizin und der Mediziner zu beeinflussen. Da eine «freie Regierung» besteht, die nur über «eingeschränkte Einkünfte» verfügt, ist der Spielraum verhältnismäßig gering. In erster Linie geht es darum, die bestehenden Einrichtungen der medizinischen Aus- und Weiterbildung dienstbar zu machen. Insbesondere soll von den Ärzten, die zum Studium nach Hause zurückkehren, verlangt werden, daß sie ein Jahr lang den in der Insel und im (Burger-)Spital diensttuenden Arzt begleiten, bevor sie zur Praxis zugelassen werden. Ein Stipendium soll einem weniger bemittelten Studenten erlauben, drei Jahre im Ausland zu reisen. Heimgekehrt, soll er den Nutzen seines Auslandaufenthaltes durch eine anatomische Demonstration oder eine Rede unter Beweis stellen.

Entsprechend ihrer Bedeutung für die medizinische Versorgung der Bevölkerung äußert sich Haller in seinem Gutachten ausführlich über die bernischen Wundärzte. Sie stellen für ihn eine Art notwendiges Übel dar, notwendig, weil sie oft mangels akademischer Ärzte die einzige Hilfe der Landbevölkerung sind, dennoch ein $\dot{U}$ bel wegen ihrer unzureichenden Ausbildung: «Ich rede von den Land Wund Ärzten nicht gerne, die von unwißenden und vermeßenen Meistern eine ähnliche Verwegenheit annehmen, und sonst weder die Anatomie, noch die Unterschiede der Krankheiten und Übel, noch die wahren Mittel jemahls zu lernen Gelegenheit haben.» ${ }^{14}$ An anderer Stelle heißt es: «wenige, sehr wenige sind aus Krankenhäusern und auß den Armeen hergenommen. Die meisten haben auf dem Lande bey einem ihnen selbst ähnlichen beruflosen, und unwißenden Meister gedient.» ${ }^{15}$ Haller forderte nun, daß auch die Wundärzte, bevor sie selbständig werden, ein Jahr in den stadtbernischen Spitälern praktizieren, und zwar nicht nur bei den chirurgisch, sondern auch bei den internistisch Kranken, 
da sie ja auf dem Lande - leider - auch innere Krankheiten behandeln müßten.

Im Gegensatz zu Haller, der den Wundarzt nur unter Aufsicht des Arztes arbeiten lassen wollte, vertrat Tissot in einem Gutachten, das er ebenfalls 1765 für den bernischen Sanitätsrat verfaßte, eine grundsätzlich andere Ansicht ${ }^{16}$. Für Chirurgen und Hebammen soll neu eine eigene Ausbildungsstätte geschaffen werden, mit Lehrstühlen, botanischem Garten und anatomischem Institut. Haller sollte nach Tissots Meinung die Leitung innehaben. Tissot akzeptierte also den Wundarzt, den Landchirurgen als einen eigenständigen medizinischen Beruf, eine Art Barfußarzt, der den Bedürfnissen der Landbevölkerung angemessen ist: die einfachen Leute haben einfache Krankheiten, für die eine einfache Medizin genügt.

Zwei Punkte in Hallers Vorschlägen verdienen näher betrachtet zu werden: die Benützung der hiesigen Spitäler und die Kontrolle der Ausbildung durch Prüfungen.

In Bern gibt es zwei Spitäler, die für den klinischen Unterricht in Frage kommen: das Burgerspital und das Inselspital. Letzteres hat 63 Betten, die im Jahresdurchschnitt von 480 Kranken belegt werden. Dieses beschränkte Krankengut ist kein Nachteil, wie Haller aus eigner Erfahrung weiß ${ }^{17}$ : «Ich bin aber versichert, daß ein mäßiges Krankenhaus allemahl lehrreicher als ein alzu beseztes, und unsäglich viel heilsamer für die Kranken ist. In Paris selbst besucht man viel lieber die Charité des hommes, als das mit Tausenden angefüllte Hotel Dieu, in welchem ein mehreres Verhältniß von Menschen stirbt, als an keinem andern Orte der Welt. Keine Anzahl von Ärzten und WundÄrzten ist fast genugsam etliche tausend Kranke mit genugsamer Aufmerksamkeit und Muße zu besorgen: und die Lufft stekt sich so gifftig mit den Dünsten der Kranken und Sterbenden an, daß selten eine Wunde ohne den kalten Brand bleibt. Zu London stirbt nicht mehr als der vieru. zwanzigste Kranke in den Hospitälern und bey unseren Krankenhäusern werden noch mehrere gerettet ... in der Insel sieht man alle Tage so wohl eine Menge schwerer inerlicher Krankheiten als plözliche Zufälle, Beinbrüche, Beinschäden, und allerley zur Wund Arzney gehöriger Handgriffe.»

Die jungen Ärzte würden in Bern den Beratungen der Aufnahmekommission im Schausaal beiwohnen und den diensttuenden Stadtarzt, den Ordinarius, auf seinen Visiten begleiten. Dieser würde «so wenig beschwert, als der mit fünfzig Schülern ins weit schlechtere Krankenhaus zu Leiden gehende Boerhaave: er könnte vielmehr von den jüngern, aber ihre Geschiklichkeit zeigenden Doctoren der Arzney, verschiedentlich in seinem schweren 
Dienste, zumahl auch im Schallenhause, erleichtert werden.» ${ }^{18}$ «Man kan leicht berechnen, wie groß der Nuzen dieses Unterrichtes sein muß, da bloß in der Insel man täglich sechzig schwere Fälle zu beobachten hätte, welches weit mehr ist, als der beliebteste Arzt jemahls auf einmal zu heilen hat ... »; in kurzer Zeit gewänne man «eben so viel Erfahrung, als in einem halben Leben sonst geschehen würde». ${ }^{19}$ Besonders wertvoll erscheint Haller dabei, daß im Spital der Therapieeffekt durch äußere Einflüsse weniger gestört werden kann, als dies in der Praxis der Fall ist, da «wegen der Volmacht des Arztes, wegen des unumschränkten Gehorsams, auch des volkommenen Außschlußes der NebenRäthe, die Erfolge der heilenden Methode gewißer wahrgenommen werden können». ${ }^{20}$

Haller ist Realist genug, sein Ziel nicht bloß durch Appelle und Vertrauen in die Einsicht des Einzelnen erreichen zu wollen. Er kennt den üblichen Minimalismus und verlangt deshalb von den Behörden eine Kontrolle der Ausbildungszeit und des Ausbildungsstandes durch Prüfungen ${ }^{21}$ : «Es ist aber bekannt, daß die Menschen ihren Zwek allemahl mit der wenigsten Mühe zu erhalten suchen, und keine Arbeit und keine Unkosten anwenden würden, sich in ihren Beruffen geschikt zu machen, wann sie beyde ersparen könnten. Es ist deswegen unumgänglich nöthig, und eine Pflicht der Obern Policey, diese Arbeit so nohtwendig zu machen, daß man ohne sie, und ohne eine genugsame Kenntniß in seiner Kunst, zur Ausübung derselben nicht gelangen, und die damit verknüpften Vortheile nicht erhalten könne. Daher sind in allen Beruffen Prüfungen angestellt worden, vom Lehrer der Gottseligkeit biß zum geringsten Handwerker. Die Prüfungen werden immer nohtwendiger, wann der gemeine Mann die Geschiklichkeit desjenigen minder zu beurtheilen im Stande ist, der einen Beruf antreten will. Es ist leicht einen schlechten Schuster von einem guten zu unterscheiden, und weit schwerer einen guten Arzt von einem andern zu erkennen, der die echte Grundlichkeit mit einigen äußerlichen Gaben ersetzt.»

Nachlässige Pflichterfüllung der Prüfenden, Geldgier und Gefälligkeit könnten den Nutzen des Examens vermindern. Deshalb soll für alle Anwärter die Mindestzeit, nach der sie sich zur Prüfung anmelden dürfen, geregelt werden. Je nach Stand sind die Anforderungen und Sanktionen unterschiedlich: die Ärzte absolvieren an der Universität eine Prüfung, «die zwar nicht so genau ist, aber dennoch die gröbste Unwißenheit ausschließt.» ${ }^{22}$ Bevor sie nicht ihr einjähriges Praktikum im Inselspital absolviert haben, sollen die Burger keine Hoffnung auf ein Stadtarztamt haben, den Angehörigen der Landstädte aber die Praxiserlaubnis in der bernischen 
Republik überhaupt verweigert werden. Die Wundärzte sind zu einer einjährigen Gehilfenzeit im Spital verpflichtet; anschließend sollen sie, da sie ja noch keine Prüfung abgelegt haben, von einer Versammlung von Ärzten und Wundärzten examiniert werden. Über den Prüfungsstoff macht Haller keine Angaben; immerhin sollen sich die Landwundärzte auch über Kenntnisse in der Behandlung der inneren Krankheiten ausweisen, da sie «wie die jezigen Umstände sind, ... in den von Stätten entblößten Gegenden die inneren Übel zu heilen haben.» ${ }^{23}$

\section{Zwischen Wissenschaft, Scharlatanismus und Vertrauen auf die Vorsehung}

Für Haller, den Forscher und akademischen Lehrer, ist es selbstverständlich, daß sich die praktische Medizin auf die Wissenschaft stützen muß. Daß sie dann erfolgreicher ist als die Volksmedizin, ist empirisch erwiesen. Die Protokolle des bernischen Sanitätsrates belegen, «daß durchgehends, so bald rechte Ärzte zu den tödlichen Seuchen beruffen werden, die Anzahl der sterbenden augenbliklich abnimt, und wo Häuser ausstarben, bloß einzelne Kranke nun mehr verlohren gehen.» ${ }^{24}$ Der Arzt darf demnach stolz sein auf seine Wissenschaft, denn die Medizin ist eines der wirksamsten Mittel gegen die Entvölkerung des Staates, jenes Übel, das die physiokratischen Patrioten des 18. Jahrhunderts auch in Bern so sehr erschreckte.

Wenn aber die Medizin dennoch nicht überall in gutem Ansehen steht, so trägt nicht zuletzt die Obrigkeit die Schuld daran. Die Ärzte sind schlecht besoldet und gezwungen, von ihren Patienten zu leben. Dadurch leidet das gegenseitige Vertrauen, die Patienten halten sich nicht an die Vorschriften und die Mittel nützen nichts. Ein Teufelskreis entsteht. Der Spitalarzt hat es hier leichter, indem der Patient äußeren Einflüssen weniger ausgesetzt ist ${ }^{25}$ : «Dann ich habe selbst in Hospitälern wahrgenommen, wie viel leichter man die völlig sich unterwerffenden Kranken heilt: Da hingegen die eigenwilligen, und durch tausend sich kreuzende Rähte irre gemachten, vornehmen Kranken überaus selten zu einer anhaltenden Beständigkeit im gebrauche der Mittel zu bereden sind, weil sie dem Arzte nur ein unvollkommenes Zutrauen gönnen.» In der freien Praxis muß der Arzt den Patienten führen können, er braucht Vertrauen, Einsicht, Gehorsam. Gerade hier fühlte Haller übrigens seine Schwäche als Praktiker. Er, der alles wußte, war nicht in der Lage, den Patienten zu überzeugen. Während der Behandlung des Schultheißen Tillier klagte er Tissot am 5. Juni $1766^{26}$ : «Je ne suis d'ailleurs 
pas fait pour insister sur mes conseils. - Je l'ai dit, et j'ai soupiré. Je ne sais pas forcer les hommes a me croire.»

Haller lehnte es entschieden ab, dem Erfolg zuliebe mit dem Scharlatanismus auch nur zu kokettieren. Er verabscheut Behandlungen, die - ob mit oder ohne Erfolg - ohne Kenntnis der Ursachen erfolgen ${ }^{27}$. So erklären sich auch seine Vorbehalte gegen Michel Schüppach, dem er zwar «genie» und "penetration» (Scharfsinn) zubilligt, der jedoch ganz unvernünftige Medikamente und überdies dieselben gegen hundert verschiedene Krankheiten gebe, und nicht einmal eine $\mathrm{W}$ aage habe, um seine Heilmittel abzuwägen, dessen wunderbare Urindiagnostik von einem Arzt entlarvt worden sei usw. ${ }^{28}$

Als selbstverständlich sieht Haller an, daß der Praktiker die Ergebnisse der Forschung zur Kenntnis nimmt und sie fortwährend in sein Wissensgebäude einbaut. Dies betrifft zum Beispiel Hallers eigene Forschungen zur Irritabilitäts- und Sensibilitätslehre, die Feststellung zum Beispiel, daß Sehnen unempfindlich und deshalb chirurgische Eingriffe an ihnen leicht möglich seien. Andere Fortschritte betreffen die Pockenimpfung, die materia medica, die chirurgische Technik. Die Lektüre, als einfachstes Mittel der Belehrung, ist hiezu Voraussetzung. Es sei erwiesen, sagen die zwei Herausgeber eines Werkes von Haller, «daß Litteratur und Belesenheit dem rechtschaffnen und würdigen Arzte äußerst nothwendig sey, daß die fähigsten Köpfe, die größten Genies auf sehr mißlichem Pfade gehen, wann sie Belesenheit verachten, und ohne sie zu Entdeckungen blos durch eigne Erfahrungen gelangen wollen; daß aber nur eine große Belesenheit, mittelmäßige Genies, worunter dann doch wohl der größre Theil der Ärzte gehört, in den Stand seze, ihren Pflichten vollkommen Genüge zu thun ....»29

Am Fortschritt der Medizin aktiv mitzuwirken, war in den Augen Hallers und seiner Zeit für den praktischen Arzt durchaus möglich und notwendig. Scharfe Beobachtung, genaue Krankengeschichten, Kombinationsgabe usw. konnten sehr wohl zu neuen Einsichten führen, man denke zum Beispiel an Witherings Beschreibung des genauen Gebrauchs der Digitalis (1785), an Jenners Kuhpockenimpfung (1798). Haller hat im Vorwort zur Pharmacopoea Helvetica sich ausführlich über die dringend notwendige Prüfung der Arzneimittel geäußert. Er hat ein ganzes Programm dafür aufgestellt, was vom behandelnden Arzt durchzuführen sei, dies mit Vorteil im Spital wegen der besseren Kontrollmöglichkeiten ${ }^{30}$.

Ein Wort schließlich zur Motivation des praktischen Arztes. Haller schildert die «Vorzüge eines gelehrten, geübten und folglich glücklichen 
Arztes» sehr lebhaft im Vorwort zu den Gedichten Werlhofs, des Dichters und Hannoverschen Leibarztes ${ }^{31}$ : «Seine Gaben sind ein Werkzeug, durch welches die Vorsehung ihre Güte ausbreitet. Erschrockene Ehegatten, zitternde Kinder, tief gerührte Ältern hoffen und erhalten öfters von ihm das erwünschte Leben eines unschätzbaren Ehmannes, einer zärtlich geliebten Frau, eines unentbehrlichen Vaters, eines hoffnungsvollen Kindes: die Sehnsucht beschleunigt seine Wege, die Hoffnung begleitet ihn, und der Segen derer, die er gerettet, folgt ihm nach, wenn er zurück geht. Ein Dichter vergnügt eine Viertelstunde, ein Arzt verbessert den Zustand eines ganzen Lebens.» Mit dichterischem Feuer malt Haller den glücklichen Arzt, sein hohes Ansehen in der menschlichen Gesellschaft, die in ihm ein Werkzeug der Vorsehung, aber auch einen nützlichen Mitbürger sieht, der die Wissenschaft zum «gemeinen Besten» und zum Wohl des einzelnen anwendet. Wahrlich Gründe genug, diesen Beruf zu ergreifen!

\section{Zusammenfassung}

Als akademischer Lehrer erwartet Haller vom Arzt seiner Zeit eine solide Ausbildung sowohl in den Grundlagenfächern der Anatomie und Physiologie als auch in der klinischen Medizin. Eigenhändige experimentelle Arbeit gehört dazu, aber auch ein längerer Aufenthalt im Spital, zum Studium von Krankheitsverläufen und Therapie aus eigener Anschauung.

Diese forschende, kritische, dem Neuem aufgeschlossene Haltung soll den Arzt auch im späteren Beruf auszeichnen, einem Beruf, der keine Halbheiten zuläßt und Überzeugungskraft und ganzen Einsatz erfordert. Als aufgeklärter Medizinalpolitiker wünscht Haller, daß die Behörden das Ansehen und die Glaubwürdigkeit der Medizin fördern durch ein Obligatorium des klinischen Unterrichts und durch eine Qualitätskontrolle mittels Prüfungen. Das Problem der medizinischen Unterversorgung des Landes möchte er durch Vermehrung der Zahl der akademisch gebildeten Ärzte gelöst sehen, nicht durch ein System von «Barfußärzten».

\section{Anmerkungen}

${ }^{1}$ Erich Hintzsche (Hrsg.), Albrecht von Hallers Briefe an Auguste Tissot, 1754-1777, Bern/ Stuttgart/Wien 1977. - Urs Boschung, Albrecht von Haller als Arzt. Zur Geschichte des Elixir acidum Halleri. Gesnerus 34, S.267-293, 1977. 
2 Johann Georg Zimmermann (Hrsg.), Über den Herrn von Haller. Aus Linguets Annalen. Übersetzt und mit Anmerkungen begleitet. Deutsches Museum, S.430-433, 1778.

${ }^{3}$ Hintzsche (Anm. 1), S. 125.

${ }^{4}$ Göttingische Anzeigen von Gelehrten Sachen, 2. Bd., S. 806-808, 1761, 1762.

${ }^{5}$ Otto Sonntag (Hrsg.), The Correspondence between Albrecht von Haller and Charles Bonnet, Bern/Stuttgart/Vienna 1983 (Studia Halleriana I), S. 1109.

${ }^{6}$ Einen kurzen Abriß von Hallers Ansichten gibt: Erich Hintzsche, A.v. Hallers «Prospectus d'un dictionnaire universel de médecine». Gesnerus 23, S. 48-54, 1966.

${ }^{7}$ Haller im Vorwort (S.4) zur Pharmacopoea helvetica ..., Basileae 1771.

${ }^{8}$ Haller in Hintzsche (Anm.6), S. 52.

${ }^{9}$ Op. cit., S. 53.

${ }^{10}$ Emil F. Rössler, Die Gründung der Universität Göttingen, Göttingen 1855, S. 301 (Gutachten von P.G. Werlhof, 16. Dez. 1733).

11 Vgl. Urs Boschung (Hrsg.), Johannes Gessners Pariser Tagebuch 1727, Bern/Stuttgart/ Toronto 1985 (Studia Halleriana II).

12 Gustav Tobler, Albrecht von Haller als bernischer Sanitätsrat. Für's Schweizer Haus 1, S.468, 484, 504, 506, 1901/1902. - Eugène Olivier, Médecine et Santé dans le Pays de Vaud au XVIII ${ }^{e}$ siècle, Lausanne 1939, Bd. 1, S. 14-17.

${ }^{13}$ Staatsarchiv Bern, B XI 132, eigene Paginierung, eingereiht zwischen S.66 und 67. Auszugsweise publiziert von Tobler (Anm.12) und Ruth Jaussi, Das Medizinische Institut in Bern (1797-1805), Bern 1944 (Berner Beiträge zur Geschichte der Medizin und der Naturwissenschaften, Nr.57), S. 6 f.

14 Hallers Gutachten (Anm. 13), S. 12.

15 Op. cit., S. 4 .

${ }^{16}$ Samuel Auguste André David Tissot, Mémoire sur les Moyens de procurer aux paysans malades les Secours les plus utiles (12 févr. 1765). Staatsarchiv Bern, B XI 132, S. 1-36. Vgl. zur Gegenüberstellung der beiden Gutachten: Urs Boschung, Médecine et Santé publique au $18^{\mathrm{e}}$ siècle à travers la correspondance d'Albert de Haller et d'Auguste Tissot. Im Druck.

17 Hallers Gutachten (Anm. 13), S. 14.

${ }_{18}$ Op. cit., S. 16

19 Ebd.

20 Ebd.

${ }^{21}$ Op. cit., S. 19 f.

${ }^{22}$ Op. cit., S. 10.

${ }^{23}$ Op. cit., S. 22.

${ }^{24}$ Op. cit., S. 9

${ }_{25}$ Op. cit., S. 7.

${ }^{26}$ Hintzsche (Anm. 1), S. 238.

27 Op. cit., S. 52.

28 Sonntag (Anm. 5), S. 1130.

29 Johann Jakob Römer, Paulus Usteri, Des Herrn von Hallers Tagebuch der medicinischen Litteratur der Jahre 1745 bis 1774. [richtig 1753], Bern 1789, Bd.1, S. IV f.

30 Pharmacopoea helvetica (Anm. 7), S.11-13.

31 Paul Gottlieb Werlhof, Gedichte ... mit einer Vorrede Herrn D. Albrecht Hallers, Hannover 1749, zit. nach Ludwig Hirzel, Albrecht von Hallers Gedichte, Frauenfeld 1882, S. 392. 


\section{Summary}

A.v. Haller's demands on the medical practitioners of his time concern both their education and practical work. The professor insists on anatomy and physiology (including research work) as the basic science of medicine. As a member of the Sanitary Council (Sanitätsrat) of Berne Haller suggests that the authorities should organize the practical education of young doctors and surgeons in the hospitals of Berne, and give travel grants to some of them. Examinations should improve the standard of medicine. Contrary to Tissot's plan to establish a new special school for surgeons as country doctors, Haller dislikes their general practice and prefers that they should be controlled by the physicians. These are expected to be interested in medical science and to contribute to its progress e.g. by discovering safe drugs.

Prof. Dr.med. Urs Boschung

Medizinhistorisches Institut der Universität

Bühlstrasse 26

3012 Bern 